\title{
AVALIAÇÃO DA SENBILIBIDADE E DA ESPECIFICIDADE DE INTERLEUCINA-29 EM PACIENTES COM ARTRITE REUMATOIDE
}

\author{
L.F. ROCHA Jr ${ }^{1}$, A. L. B. P. DUARTE ${ }^{1}$, M. J. B. M. RÊGO ${ }^{2}$, A. B. F. PAIXÃO ${ }^{2}$, K. M.

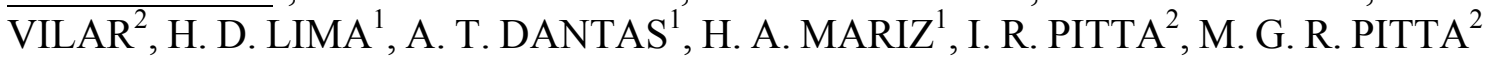 \\ ${ }^{1}$ Hospital das Clínicas da Universidade Federal de Pernambuco (HC UFPE), Serviço de \\ Reumatologia; \\ ${ }^{2}$ Universidade Federal de Pernambuco (UFPE), Departamento de Bioquímica, Laboratório \\ de Imunomodulação e Novas Abordagens Terapêuticas (LINAT). \\ E-mail para contato: laurindorochajr@gmail.com
}

\begin{abstract}
RESUMO - A interleucina-29 (IL-29) foi identificada na artrite reumatoide (AR) e relatada como um biomarcador da doença. O objetivo deste trabalho foi analisar a precisão da IL-29 na AR, em indivíduos normais e em pacientes com outras doenças reumatológicas. Os níveis séricos de IL-29 foram investigados por ELISA. Foram calculados os indices de sensibilidade, especificidade e verossimilhança (LR) para o diagnóstico de AR. Os níveis médios de IL-29 foram aumentados em pacientes com AR 113,6 (IQR = 31,25-308,5) $\mathrm{pg} / \mathrm{ml} \mathrm{em}$ comparação com pacientes com outras doenças $31,25 \mathrm{pg} / \mathrm{ml}$ e controles saudáveis 31,25 pg/ml, $p<0,0001$. Os valores de corte de IL-29 para distinguir pacientes com AR de outros pacientes pacientes foram $61,11 \mathrm{pg} / \mathrm{ml}$ (sensibilidade 57,02, especificidade 92,71, LR: 7,82) e para todos os individuos foi de 32,96 pg / ml (sensibilidade 64,46, especificidade 87,31, LR: 5.08). Níveis séricos de IL-29 é maior em pacientes com AR em comparação com outros indivíduos e pode ser usado como marcador biológico potencial.
\end{abstract}

PALAVRAS-CHAVE: Precisão, diagnóstico, interleucina-29.

ABSTRACT: Interleukin-29 (IL-29) was identified in rheumatoid arthritis (RA) and reported as a biomarker of the disease. The objective of this study was to analyze the accuracy of IL-29 in RA, in normal individuals and in patients with other rheumatic diseases. Serum levels of IL-29 were investigated by ELISA. Sensitivity, specificity and likelihood (RA) indexes for RA were calculated. Mean levels of IL-29 were increased in patients with RA $113.6(I Q R=31.25-308.5) \mathrm{pg}$ $/ \mathrm{ml}$ compared to non-RA patients $31.25 \mathrm{pg} / \mathrm{ml}$ and healthy controls $31.25 \mathrm{pg} / \mathrm{ml}$, $p<0.0001$. The cutoff values of IL-29 to distinguish $R A$ patients from non-RA patients were $61.11 \mathrm{pg} / \mathrm{ml}$ (sensitivity 57.02, specificity 92.71, LR: 7.82) and for all subjects it was 32,96 pg / $\mathrm{ml}$ (sensitivity 64.46, specificity 87.31, LR: 5.08). IL29 serum is higher in the serum of $R A$ patients compared to non-RA subjects and can be used as a potential biological marker.

KEYWORDS: Accuracy,diagnosis, interleukin-29. 


\section{INTRODUÇÃO}

A artrite reumatoide (AR) é uma doença autoimune sistêmica crônica caracterizada por inflamação sinovial e, às vezes, com características extra-articulares. As citocinas têm um papel fundamental em doenças autoimunes como a AR e estão diretamente implicadas em muitos processos associados à patogênese da doença (Mcinnes e Schett, 2011). A interleucina-29 (IL-29) revelou-se com potencial função imunorreguladora, uma vez que inibe as respostas Th2 humanas e tem atividade moduladora na resposta Th1 / Th2 (Jordan et al., 2007; Srinivas et al., 2008).

O efeito modificador da IL-29 na inflamação mediada por TLR4 (do inglês, toll-like receptor) pode contribuir com a patogênese da RA, uma vez que TLR4 é importante para o reconhecimento e ativação de patógenos do sistema imune inato (Xu et al., 2015). As citocinas demonstraram ser biomarcadores na AR e podem auxiliar no diagnóstico diferencial e proporcionar benefícios adicionais na classificação dos subtipos da doença (Trouw e Mahler, 2012; Burska et al., 2014). Com base nesses achados, o objetivo deste estudo foi avaliar a precisão da IL-29 na AR em comparação com outras doenças reumáticas e indivíduos saudáveis.

\section{MATERIAIS E MÉTODOS}

Um total de 121 pacientes com AR (11 homens, 110 mulheres, média de idade 52,9 anos, variação de 22-79 anos) foram recrutados no Ambulatório de Reumatologia do Hospital das Clínicas da Universidade Federal de Pernambuco (UFPE). Os indivíduos com outras doenças reumatológicas incluíram 60 pacientes com esclerose sistêmica (ES), 53 pacientes com lúpus eritematoso sistêmico (LES), 50 pacientes com osteoartrite (OA) e 29 pacientes com fibromialgia (FM). Sessenta e oito indivíduos saudáveis (IS) foram incluídos como controles, todos eles não apresentavam nenhuma doença reumatológica e não estavam em uso de nenhum medicamento nas últimas duas semanas.

As citocinas nos soros foram mensuradas com o kit ELISA de acordo com a recomendação do fabricante ( $\mathrm{R} \& \mathrm{D}$ Systems). O limite inferior de detecção para o kit ELISA IL-29 foi de $31,25 \mathrm{pg} / \mathrm{ml}$. Todos os ensaios foram realizados em duplicata. Os resultados foram apresentados como intervalo mediano e interquartil. As associações de níveis séricos de IL-29 com parâmetros clínicos e laboratoriais dos pacientes foram analisadas por comparações univariadas utilizando o teste de Mann-Whitney para dados não-paramétricos e teste $t$ de Student para dados paramétricos. A correlação entre as características clínicas e laboratoriais e a IL-29 sérica foi analisada utilizando o teste de correlação de classificação de Spearman para distribuição não normal e o teste de correlação de Pearson para distribuições normais.

\section{RESULTADOS E DISCUSSÃO}

Os níveis medianos de IL-29 no soro foram significativamente aumentados em pacientes com AR 113,6 pg/ml (IQR = 31,25-308,5) em comparação com ES 31,25 pg/ml, com OA 31,25 pg/ml, com FM 31,25 pg/ml e indivíduos saudáveis (IS) 31,25 pg / ml, p $<0,0001$ para todos eles. Os níveis medianos de pacientes com AR também foram aumentados em comparação com pacientes com LES 31,25 (IQR = 31,25-57,04) pg/ml, p =0,0001 (Figura 1). Quando os níveis séricos de RA (mediana 113.6) foram comparados a todos os indivíduos estudados (mediana 31.25pg / ml) e com pacientes com outras doenças (mediana $31.25 \mathrm{pg} /$ $\mathrm{ml}$ ), a análise também foi significante $(\mathrm{p}<0.0001)$. Pacientes com ES, OA, FM e controles 
saudáveis tiveram níveis médios de IL-29 no soro abaixo do limite inferior de detecção na análise.

Figura 1 - Níveis de IL-29 no soro de pacientes com artrite reumatoide (AR), pacientes com outras doenças reumatológicas (não-AR) e controles saudáveis (IS). **** $\mathrm{p}<0,0001$ para AR vs Esclerose Sistêmica (SSc), Fibromialgia (FM), Osteoartrite (OA), NHS, pacientes não-AR e em geral; ${ }^{* *} \mathrm{p}=0,0001$ para AR versus Lúpus Eritematoso Sistêmico (LES).

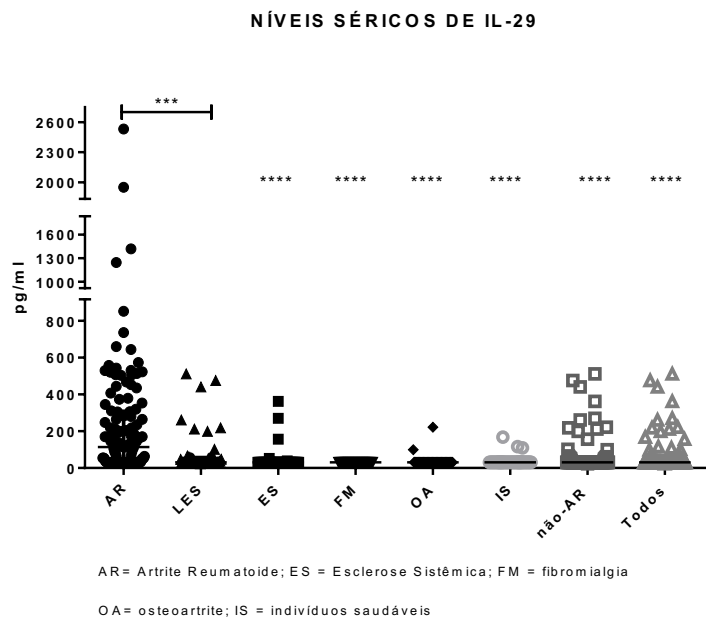

Em seguida, investigamos a sensibilidade e especificidade da IL-29 em identificar AR entre as outras doenças reumatológicas e entre controles saudáveis. A área sob a curva (AUC, do inglês area under the curve) da análise ROC foi 0,672 (CI 0,597-0,742) para IL-29 na distinção de RA de LES, 0,793 (CI 0,727-0,850) de SSc, 0,810 (CI 0,743-0,866) de OA, 0,826 (CI 0,756-0,883) de FM e 0.810 (CI 0.747-0.864) de controles saudáveis. Considerando que, quando tomados em conjunto, todos os pacientes que não têm o diagnóstico de $\mathrm{AR}$, a $\mathrm{AUC}$ foi 0,769 (CI 0,719-0,815). Enquanto isso, o desempenho da IL-29 sérica na AR versus todos os indivíduos do estudo demonstrou uma AUC da análise ROC de 0.780 (CI 0.735-0.821) (Figura 2). Ao usar o corte de IL-29 de $32,96 \mathrm{pg} / \mathrm{ml}$ para identificar a AR em todos os indivíduos analisados, a porcentagem de pacientes corretamente classificados foi de $64,46 \%$ (sensibilidade) e a porcentagem classificada corretamente como não tendo RA foi de $87,31 \%$ (especificidade).

Figura 2 - Sensibilidade e especificidade da IL-29 em identificar Artrite Reumatoide (AR) entre outras doenças reumatológicas e controles saudáveis. AUC (Área sobre a curva, do inglês area under the curve); CI (Intervalo de confiança, do inglês confidence interval).

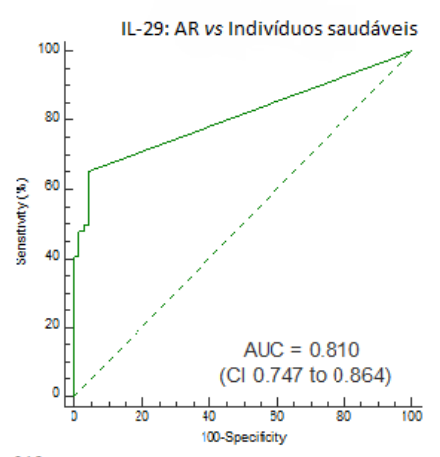

[A]

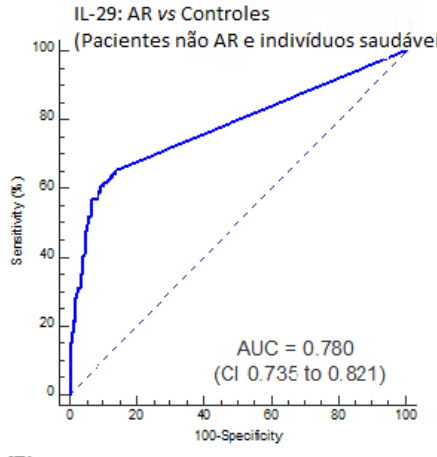

[B]

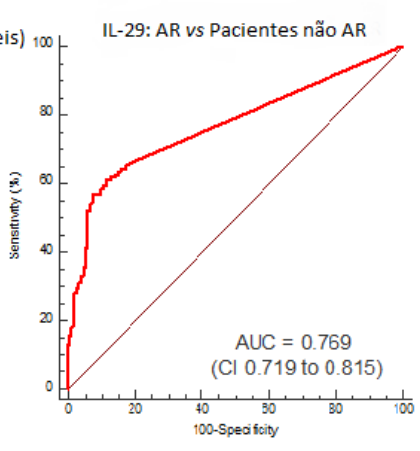

$[\mathrm{C}]$

Com base nos achados acima, o presente estudo demonstrou que a IL-29 poderia ser um 
marcador de laboratório útil para discriminação entre pacientes com $\mathrm{AR}$ e pacientes com outras doenças reumatológicas, bem como pacientes com AR e indivíduos saudáveis. Este foi o primeiro estudo a demonstrar níveis mais altos de IL-29 na artrite reumatoide entre pacientes com outras doenças reumatológicas e a performance destes na identificação de pacientes com AR entre indivíduos com doenças que podem apresentar queixas articulares.

\section{CONCLUSÃO}

Nossos resultados sugerem que a IL-29 pode ser um biomarcador nas doenças autoimunes especialmente na AR e que os níveis séricos de IL-29 podem ser usados como um marcador de diagnóstico biológico na AR. Para o nosso conhecimento, este é o primeiro estudo a demonstrar a capacidade da IL-29 para a diferenciação entre pacientes com AR e indivíduos com outras doenças reumáticas. Esses achados sugerem que medir os níveis de IL29 pode ser uma ferramenta útil para avaliar a presença de AR. Outros estudos são necessários para elucidar o potencial do desempenho diagnóstico de IL-29 na AR, especialmente com pacientes em fases iniciais da doença.

\section{REFERENCIAS}

BURSKA, A.; BOISSINOT, M.; PONCHEL, F. Cytokines as biomarkers in rheumatoid arthritis. Mediators Inflamm, v. 2014, p. 545493, 2014. ISSN 1466-1861. Disponível em: < https://www.ncbi.nlm.nih.gov/pubmed/24733962 >.

JORDAN, W. J. et al. Human interferon lambda-1 (IFN-lambda1/IL-29) modulates the Th1/Th2 response. Genes Immun, v. 8, n. 3, p. 254-61, Apr 2007. ISSN 1466-4879. Disponível em: < https://www.ncbi.nlm.nih.gov/pubmed/17361203 >.

MCINNES, I. B.; SCHETT, G. The pathogenesis of rheumatoid arthritis. N Engl J Med, v. 365, n. 23, p. 2205-19, Dec 2011. ISSN 1533-4406. Disponível em: < https://www.ncbi.nlm.nih.gov/pubmed/22150039>.

SRINIVAS, S. et al. Interferon-lambda1 (interleukin-29) preferentially down-regulates interleukin-13 over other T helper type 2 cytokine responses in vitro. Immunology, v. 125, n. 4, p. 492-502, Dec 2008. ISSN 0019-2805.

TROUW, L. A.; MAHLER, M. Closing the serological gap: promising novel biomarkers for the early diagnosis of rheumatoid arthritis. Autoimmun Rev, v. 12, n. 2, p. 318-22, Dec 2012. ISSN 1873-0183. Disponível em: < https://www.ncbi.nlm.nih.gov/pubmed/22664776 >.

XU, D. et al. IL-29 Enhances LPS/TLR4-Mediated Inflammation in Rheumatoid Arthritis. Cell Physiol Biochem, v. 37, n. 1, p. 27-34, 2015. ISSN 1421-9778. Disponível em: < https://www.ncbi.nlm.nih.gov/pubmed/26278073 $>$.

\section{AGRADECIMENTOS}

INCT_if ; FACEPE - Fundação de Amparo à Ciência e Tecnologia do Estado de Pernambuco 